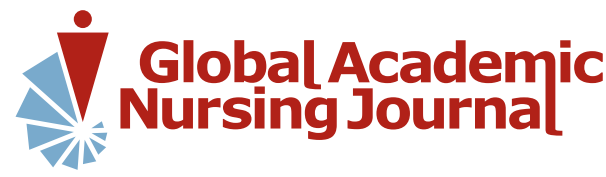

\section{Estilo de vida e adesão à terapêutica num grupo de pessoas portadoras de hipertensão arterial}

\author{
Lifestyle and adherence to therapy in a group of people with arterial hypertension
}

Estilo de vida y adherencia a la terapia en un grupo de personas con hipertensión arterial

\section{Andrea Silva Santos Pinto ${ }^{1}$ ORCID: 0000-0001-5529-0285 \\ Ermelinda Maria Gonçalves Bernardo Marques ${ }^{2}$ ORCID: 0000-0003-3024-8392 \\ Dora Maria Ricardo Fonseca Saraiva $^{3}$ \\ ORCID: 0000-0002-2518-3371}

${ }^{1}$ Unidade de Cuidados de Saúde Personalizados. Teixoso,

Portugal.

${ }^{2}$ Escola Superior de Saúde do Instituto Politécnico da Guarda. Guarda, Portugal.

${ }^{3}$ Centro Hospitalar Universitário Cova da Beira. Covilhã, Portugal.

\section{Como citar este artigo:}

Pinto ASS, Marques EMGB, Saraiva DMRF. Estilo de vida e adesão à terapêutica num grupo de pessoas portadoras de hipertensão arterial. Glob Acad Nurs. 2021;2(3):e149. https://dx.doi.org/10.5935/26755602.20200149

Autor correspondente: Andrea Silva Santos Pinto E-mail: andreass.enf@gmail.com

Editor Chefe: Caroliny dos Santos Guimarães da Fonseca Editor Executivo: Kátia dos Santos Armada de Oliveira

Submissão: 01-06-2021 Aprovação: 20-07-2021

\section{Resumo}

Objetivou-se avaliar o estilo de vida e a adesão à terapêutica num grupo de pessoas portadoras de HTA, numa Unidade de Cuidados de Saúde Personalizados. É um estudo quantitativo, descritivo e transversal. A amostra é de 314 clientes. Utilizou-se um protocolo de avaliação, com as escalas: Medida de Adesão aos Tratamentos, Escala de Hábitos Alimentares, Alcohol Use Disorders Identification Test, o Teste de Fagerström de Dependência à Nicotina e o Questionário Internacional de Atividade Física versão curta. Resultados revelam que $88,2 \%$ dos inquiridos aderem à terapêutica prescrita. No estilo de vida, constata-se que $96,2 \%$ dos clientes têm hábitos alimentares adequados; 94,3\% têm baixa probabilidade de consumir bebidas alcoólicas, 5,1\% são fumadores e 49,4\% são "insuficientemente ativos". Os resultados sugerem a necessidade de intervir na promoção da saúde através de um programa comunitário que vise a adesão à terapêutica e capacite a pessoa na adoção de um estilo de vida saudável.

Descritores: Estilo de Vida; Adesão à Medicação; Adesão do Paciente; Adesão e Adesão ao Tratamento; Hipertensão.

\begin{abstract}
The aim was to evaluate the lifestyle and adherence to therapy in a group of people with HTA, in a Personalized Health Care Unit. It is a quantitative, descriptive, and cross-sectional study. The sample is from 314 customers. An assessment protocol was used, with the following scales: Treatment Compliance Measure, Eating Habits Scale, Alcohol Use Disorders Identification Test, the Fagerström Nicotine Dependence Test and the International Physical Activity Questionnaire, short version. Results show that $88.2 \%$ of respondents adhere to the prescribed therapy. In terms of lifestyle, it appears that $96.2 \%$ of customers have adequate eating habits; $94.3 \%$ are unlikely to consume alcoholic beverages, $5.1 \%$ are smokers and $49.4 \%$ are "insufficiently active". The results suggest the need to intervene in health promotion through a community program aimed at adherence to therapy and empowering the person to adopt a healthy lifestyle.
\end{abstract}

Descriptors: Lifestyle; Medication Adherence; Patient Compliance; Treatment Adherence and Compliance; Hypertension.

\section{Resumén}

El objetivo fue evaluar el estilo de vida y la adherencia a la terapia en un grupo de personas con HTA, en una Unidad de Atención Personalizada. Es un estudio cuantitativo, descriptivo y transversal. La muestra es de 314 clientes. Se utilizó un protocolo de evaluación, con las siguientes escalas: Medida de Adherencia al Tratamiento, Escala de Hábitos Alimenticios, Test de Identificación de Trastornos por Uso de Alcohol, Test de Dependencia de Nicotina Fagerström y Cuestionario Internacional de Actividad Física, versión corta. Los resultados muestran que el $88,2 \%$ de los encuestados se adhieren a la terapia prescrita. En términos de estilo de vida, parece que el $96,2 \%$ de los clientes tienen hábitos alimentarios adecuados; El 94,3\% tiene poca probabilidad de consumir bebidas alcohólicas, el $5,1 \%$ son fumadores y el $49,4 \%$ son "insuficientemente activos". Los resultados sugieren la necesidad de intervenir en la promoción de la salud a través de un programa comunitario dirigido a la adherencia a la terapia y empoderar a la persona para que adopte un estilo de vida saludable.

Descriptores: Estilo de Vida; Adherencia a la Medicación; Cumplimiento del Paciente; Adherencia y Cumplimiento de Tratamiento; Hipertensión. 


\section{Introdução}

A Hipertensão Arterial (HTA) tem uma elevada prevalência em todo o mundo, constituindo um grave problema para a saúde pública ${ }^{1}$.

Portugal é um dos países da Europa com uma das mais elevadas taxas de HTA. Concretizando, na Cova da Beira a situação é similar, a HTA sem complicações é a segunda morbilidade mais prevalente ${ }^{2}$. A sua elevada prevalência conduz a avultosos custos económicos, a nível individual e social, pelo que se torna urgente o seu estudo.

Para minimizar os seus efeitos, é necessário intervir no controlo dos fatores de risco modificáveis, através da adoção de um estilo de vida saudável e na otimização do regime terapêutico ${ }^{3,4}$.

Em Portugal as taxas de adesão à terapêutica oscilam entre os $16,9 \%$ e os $91,3 \%{ }^{5,6}$.

A adesão à terapêutica pode ser definida como a correta execução da prescrição farmacológica já que existem diversos fatores que influenciam a adesão da mesma. Os principais fatores podem ser agrupados em: i) fatores sociais, económicos e culturais; ii) fatores relacionados com os profissionais e os serviços de saúde; iii) fatores relacionados com a doença de base e as comorbilidades; iv) fatores relacionados com a terapêutica prescrita e v) fatores individuais relativos ao cliente $\mathrm{c}^{7-12}$.

A não adesão à terapêutica tem consequências a nível da qualidade de vida, sendo uma das principais causas do controlo inadequado da pressão arterial aumentando, deste modo, o risco de complicações associadas ${ }^{7,10,11,13}$.

Isto reforça o papel preponderante do enfermeiro no desenvolvimento de estratégias promotoras da adesão à terapêutica e de um estilo de vida saudável.

A definição de estilo de vida surge no âmbito de várias estratégias promotoras da saúde, que envolve determinados comportamentos feitos a partir de diferentes escolhas dos indivíduos e, são condicionadas pelas oportunidades de vida ${ }^{14}$. Neste âmbito, os organismos governamentais têm desenvolvido vários programas que visam a promoção de um estilo de vida saudável, a nível mundial.

Neste sentido, considera-se pertinente estudar esta temática, por um lado a HTA, é considerada uma das mais graves doenças da atualidade, constituindo-se um dos principais fatores de risco das doenças cardiovasculares; por outro lado, a adesão à terapêutica é muito importante no controlo da HTA, o estilo de vida saudável é uma das medidas a adotar no tratamento não farmacológico desta.

A solidez e consistência desta pesquisa terão certamente como consequência a conquista de novas estratégias de intervenção da Enfermagem.

O objetivo geral deste estudo consiste em avaliar a adesão à terapêutica e estilo de vida, num grupo de pessoas portadoras de HTA, numa Unidade de Cuidados de Saúde Personalizados (UCSP), da Região Centro de Portugal. Foi constituída uma questão de investigação: Qual a adesão à terapêutica e estilo de vida num grupo de pessoas portadoras de HTA?

\section{Metodologia}

Trata-se de uma investigação não experimental, com abordagem quantitativa. É um estudo descritivo, analítico, correlacional e transversal.

A população alvo do estudo é constituída por 1688 clientes inscritos no "PNDCC Risco: Hipertensão" e seguidos na consulta de HTA, de uma UCSP da Região Centro de Portugal. A técnica de amostragem foi não probabilística, acidental. No cálculo do tamanho amostral utilizou-se o programa informático Raosoft, de forma a garantir a representatividade da população, tendo sido estudados 314 clientes.

Foi utilizado um protocolo de avaliação de héteropreenchimento, aplicado durante uma entrevista. Este protocolo inclui questões relacionadas com o perfil sociodemográfico; socioprofissional; dados antropométricos e dados clínicos ${ }^{15}$.

A adesão à terapêutica foi avaliada através da Medida de Adesão aos Tratamentos (MAT), validada por Delgado e Lima $^{16}$ para a população portuguesa e permite avaliar em que medida o cliente segue as orientações fornecidas pelos profissionais de saúde, bem como os seus comportamentos quanto à adesão terapêutica.

Os dados referentes ao estilo de vida foram obtidos através de quatro escalas, sobre: hábitos alimentares, o consumo de álcool, os hábitos tabágicos e a atividade física.

Os hábitos alimentares foram avaliados através da utilização da Escala de Hábitos Alimentares (EHA). A EHA é composta de enunciados, os seus quarenta itens estão agrupados em quatro dimensões: "quantidade alimentar", "qualidade alimentar"," variedade alimentar" e "adequação alimentar"17.

O consumo de álcool foi avaliado através do Alcohol Use Disorders Identification Test (AUDIT), desenvolvido pela WHO. O AUDIT é constituído por dez itens, agrupados em três dimensões ${ }^{18}$ relativas a: "caraterização do consumo", "sintomas de dependência" e "consequências do consumo". Relativamente aos hábitos tabágicos foi utilizado o Teste de Fagerström de Dependência à Nicotina, como medida de avaliação da dependência tabágica. A versão portuguesa foi validada por estudo ${ }^{21}$ e é constituído por seis itens relativos à dependência da nicotina.

Para avaliar a atividade física foi utilizado Questionário Internacional de Atividade Física (IPAQ), versão curta, cuja versão portuguesa foi validada por Campaniço. $O$ IPAQ foi proposto pela WHO, como um instrumento mundial para determinar o nível de atividade física da população, sendo o instrumento mais amplamente utilizado a nível mundial para avaliar atividade física. Na versão curta o IPAQ tem nove itens, com questões abertas que permitem avaliar o comportamento referente à atividade física e ao sedentarismo nos últimos 7,0 dias ${ }^{19}$.

A colheita de dados foi realizada entre 27 de maio a 15 de julho de 2019 , após a aplicação de um pré-teste. A análise dos dados foi realizada através do programa Statistical Package for Social Sciences (SPSS), versão 25.0. 
A investigação cumpriu todos os procedimentos éticos/legais. Os clientes que participaram neste estudo foram previamente esclarecidos que os dados obtidos seriam mantidos em sigilo e privacidade, foi assinado o consentimento livre e esclarecido, segundo a Resolução $n$. o 466/12 do Conselho Nacional de Saúde. Foram obtidas a autorização do Conselho Clínico e o parecer do Conselho de Ética, referente à Unidade onde foi realizado o estudo.

\section{Resultados e Discussões}

Em termos sociodemográficos, a maioria dos clientes pertence ao sexo feminino (63,7\%). A média da idade é de 68,8 anos, tendo o indivíduo mais novo 25 anos e o mais velho 89 anos. A maioria é casado (72,0\%) e reside em meio urbano (68,5\%).

Relativamente às habilitações literárias, 53,2\% possuem o 4을 ano de escolaridade, $75,8 \%$ são reformados, $51,3 \%$ possuem um rendimento no escalão menos de $419 €$ e $42,7 \%$ consideram que o seu rendimento é suficiente para as despesas com a medicação.

Em relação à caraterização antropométrica da amostra, verifica-se que $41,4 \%$ dos clientes são pré-obesos, sendo os homens $(46,5 \%)$ os que mais contribuíram para esta percentagem.

Quanto ao risco metabólico, foi feita uma avaliação do perímetro abdominal que demonstra que ambos os sexos apresentam risco muito aumentado de complicações metabólicas.
Na caraterização clínica, verifica-se que $30,6 \%$ dos inquiridos tem HTA grau I ou ligeira e 22,9\% fazem uma vigilância semanal da pressão arterial. Os clientes mencionam a presença de outras doenças, maioritariamente $(67,5 \%)$ as endócrinas, nutricionais, metabólicas e imunológicas. A maioria $(46,5 \%)$ realizou 1 consulta de enfermagem de HTA, no último ano. Em média os clientes tomam 1,2 comprimidos diferentes para a HTA e 93,9\% tomam outro tipo de medicação, a maioria $(96,2 \%)$ medicamentos para o aparelho cardiovascular. No que se refere à autoadministração da medicação, 94,6\% dos inquiridos são autónomos e não necessitam de ajuda.

Relativamente à classificação da adesão à terapêutica, conforme se pode visualizar na Tabela 1, 88,2\% dos inquiridos aderem à terapêutica, contudo $11,8 \%$ não aderem à terapêutica prescrita. No que se refere ao sexo, verifica-se que os homens $(91,2 \%)$ aderem em maior número que as mulheres $(86,5 \%)$.

Estes resultados podem ser explicados pelo facto de a amostra ser constituída por indivíduos com baixa escolaridade, e como consequência, níveis baixos de literacia. Por isso nas consultas podem apresentar níveis elevados de ansiedade e crenças erradas sobre a terapêutica. Resultados similares são os encontrados num estudo na Nigéria, em que a adesão à terapêutica com antihipertensores encontra-se nos $90,0 \%^{8}$.

Tabela 1. Classificação da adesão à terapêutica dos clientes por sexo. Teixoso, Portugal, 2019

\begin{tabular}{|c|c|c|c|c|c|c|c|}
\hline \multirow{3}{*}{\multicolumn{2}{|c|}{ Adesão à terapêutica }} & \multicolumn{4}{|c|}{ Sexo } & \multirow{2}{*}{\multicolumn{2}{|c|}{ Total }} \\
\hline & & \multicolumn{2}{|c|}{ Feminino } & \multicolumn{2}{|c|}{ Masculino } & & \\
\hline & & $\mathbf{n}$ & $\%$ & $\mathbf{n}$ & $\%$ & $\mathbf{n}$ & $\%$ \\
\hline & Não adesão & 27 & 13,5 & 10 & 8,8 & 37 & 11,8 \\
\hline & Adesão & 173 & 86,5 & 104 & 91,2 & 277 & 88,2 \\
\hline \multicolumn{2}{|c|}{ Total } & 200 & 100,0 & 114 & 100,0 & 314 & 100,0 \\
\hline
\end{tabular}

O estilo de vida foi avaliado através das escalas EHA, o AUDIT, o Teste de Fagerström de Dependência à Nicotina e o IPAQ versão curta.

No que concerne à classificação dos hábitos alimentares dos inquiridos, conforme se pode observar na Tabela 2, a maioria dos clientes $(96,2 \%)$ têm hábitos alimentares adequados, contrapondo, os 3,8\% com hábitos desadequados. Em relação ao sexo, verifica-se que quase a totalidade dos homens $(99,1 \%)$ têm hábitos alimentares adequados e $94,5 \%$ das mulheres também. Ainda que neste estudo, os hábitos alimentares desadequados sejam pouco evidentes, em Portugal, contribuem para que $50,0 \%$ dos adultos apresentem excesso de peso ${ }^{20}$.

Tabela 2. Classificação dos hábitos alimentares dos clientes por sexo. Teixoso, Portugal, 2019

\begin{tabular}{|c|c|c|c|c|c|c|}
\hline \multirow{2}{*}{ Hábitos alimentares } & \multicolumn{4}{|c|}{ Sexo } & \multicolumn{2}{|c|}{ Total } \\
\cline { 2 - 8 } & Feminino & \multicolumn{2}{|c|}{ Masculino } & \multicolumn{2}{|c|}{} \\
\cline { 2 - 8 } & $\mathbf{n}$ & $\mathbf{\%}$ & $\mathbf{n}$ & $\mathbf{\%}$ & $\mathbf{n}$ & $\%$ \\
\hline Hábitos desadequados & 11 & 5,5 & 1 & 0,9 & 12 & 3,8 \\
\hline Hábitos adequados & 189 & 94,5 & 113 & 99,1 & 302 & 96,2 \\
\hline Total & 200 & 100,0 & 114 & 100,0 & 314 & 100,0 \\
\hline
\end{tabular}

Relativamente à classificação do consumo de álcool dos inquiridos, conforme se pode observar na Tabela 3, a maioria dos clientes $(94,3 \%)$, têm baixa probabilidade de consumir bebidas alcoólicas. No entanto, 5,1\% têm risco e $0,6 \%$ têm um consumo nocivo de álcool.

Tabela 3. Classificação do consumo de álcool dos clientes por sexo. Teixoso, Portugal, 2019

\begin{tabular}{|c|c|c|c|c|c|c|}
\hline \multirow{2}{*}{ Consumo de álcool } & \multicolumn{4}{|c|}{ Sexo } & \multicolumn{2}{c|}{ Total } \\
\cline { 2 - 7 } & \multicolumn{2}{|c|}{ Feminino } & Masculino & \multicolumn{2}{c|}{} \\
\cline { 2 - 7 } & $\mathbf{n}$ & $\%$ & $\mathbf{n}$ & $\%$ & $\mathbf{n}$ & $\%$ \\
\hline Dependência & 0 & 0,0 & 0 & 0,0 & 0 & 0,0 \\
\hline
\end{tabular}




\begin{tabular}{|c|c|c|c|c|c|c|}
\hline Consumo nocivo & 0 & 0,0 & 2 & 1,8 & 2 & 0,6 \\
\hline Tem risco & 0 & 0,0 & 16 & 14,0 & 16 & 5,1 \\
\hline Baixa probabilidade & 200 & 100,0 & 96 & 84,2 & 296 & 94,3 \\
\hline Total & 200 & 100,0 & 114 & 100,0 & 314 & 100,0 \\
\hline
\end{tabular}

A prevalência de consumo de tabaco neste estudo é de $5,1 \%$, sendo que $2,0 \%$ são mulheres e $10,5 \%$ são homens.

A classificação dos hábitos tabágicos, pode observar-se na Tabela 5. Dos clientes com hábitos tabágicos, a maioria $(62,4 \%)$, tem "dependência baixa" à nicotina, porém, em igual percentagem, $18,8 \%$ dos clientes, têm uma "dependência alta" e uma "dependência média" à nicotina. Fazendo a análise por sexo verifica-se que a "dependência alta" acontece apenas nos homens $(25,0 \%)$, tendo mulheres uma percentagem nula (0,0\%). Porém são, maioritariamente, as mulheres $(25,0 \%)$ que apresentam uma "dependência média", comparativamente aos homens $(16,7 \%)$. Também são as mulheres, em maior percentagem, a apresentar uma "dependência baixa" (75,0\%), em relação aos homens (58,3\%).

Neste âmbito, é importante que o enfermeiro incentive a cessação tabágica, e faça o encaminhamento do cliente para a respetiva consulta de cessação tabágica, bem como para a página web do Programa de Capacitação para Cessação Tabágica, do hospital de referência.

Tabela 4. Classificação dos hábitos tabágicos dos clientes por sexo. Teixoso, Portugal, 2019
\begin{tabular}{|c|c|c|c|c|c|c|}
\hline \multirow{2}{*}{ Hábitos tabágicos } & \multicolumn{3}{|c|}{ Sexo } & \multicolumn{2}{|c|}{ Total } \\
\cline { 2 - 7 } & Feminino & Masculino & \multicolumn{2}{c|}{} \\
\cline { 2 - 8 } & $\mathbf{n}$ & $\%$ & $\mathbf{n}$ & $\%$ & $\mathbf{n}$ & $\%$ \\
\hline Dependência alta & 0 & 0,0 & 3 & 25,0 & 3 & 18,8 \\
\hline Dependência média & 1 & 25,0 & 2 & 16,7 & 3 & 18,8 \\
\hline Dependência baixa & 3 & 75,0 & 7 & 58,3 & 10 & 62,4 \\
\hline Total & 4 & 100,0 & 12 & 100,0 & 16 & 100,0 \\
\hline
\end{tabular}

Fazendo a classificação da atividade física segundo - IPAQ versão curta, pode visualizar-se na Tabela 6 que 49,4\% dos clientes são "insuficientemente ativos" ou sedentários, todavia $29,6 \%$ são "vigorosamente ativos" e 21,0\% são "moderadamente ativos".

Estes dados vão ao encontro da literatura, em que os clientes com HTA, revelam uma baixa adesão à atividade física. Outras investigações demonstram que $47,0 \%$ dos inquiridos apresentam uma atividade física baixa ${ }^{21}$.

Em consonância, um estudo ${ }^{22}$ revela que $21,0 \%$ dos clientes praticam regularmente exercício físico.

A baixa adesão à atividade física poderá ser devido ao facto de a maioria dos inquiridos serem idosos e terem problemas osteoarticulares tornando os clientes mais limitados fisicamente. A falta de recursos na comunidade que estimulem a atividade física, poderá constituir um fator condicionante.

Contudo, nas zonas rurais os idosos têm a oportunidade de serem mais ativos, pois é comum terem alguma atividade ligada à agropecuária. Assim, é importante que o enfermeiro incentive os clientes a praticarem exercício físico, adequado a cada situação específica, designadamente a realização de caminhadas ou subir escadas (um lance de escadas equivale a 20 degraus e corresponde a 10 minutos de caminhada).

Tabela 5. Classificação da atividade física dos clientes por sexo. Teixoso, Portugal, 2019

\begin{tabular}{|c|c|c|c|c|c|c|}
\hline \multirow{2}{*}{ Atividade física } & \multicolumn{4}{|c|}{ Sexo } & \multicolumn{2}{|c|}{ Total } \\
\cline { 2 - 6 } & \multicolumn{2}{|c|}{ Feminino } & \multicolumn{2}{c|}{ Masculino } & \multicolumn{2}{|c|}{} \\
\cline { 2 - 6 } & $\mathbf{n}$ & $\mathbf{\%}$ & $\mathbf{n}$ & $\mathbf{n}$ & $\mathbf{n}$ & $\%$ \\
\hline Insuficientemente ativo & 111 & 55,5 & 44 & 38,5 & 155 & 49,4 \\
\hline Moderadamente ativo & 42 & 21,0 & 24 & 21,1 & 66 & 21,0 \\
\hline Vigorosamente ativo & 47 & 23,5 & 46 & 40,4 & 93 & 29,6 \\
\hline Total & 200 & 100,0 & 114 & 100,0 & 314 & 100,0 \\
\hline
\end{tabular}

No que concerne às limitações, encontra-se o fato deste estudo apenas se restringir aos clientes de uma UCSP. Outro aspeto a destacar, em termos metodológicos é a utilização do método de amostragem não probabilístico. Embora seja fácil e rápido, os resultados apenas se referem à amostra utilizada, não podendo ser extrapolados com confiança para a restante população. Daí que seja necessária a confirmação destes resultados com amostras mais abrangentes e representativas.

\section{Conclusão}

A revisão bibliográfica indicou que a HTA devido à elevada prevalência e incidência, constitui um grave problema de saúde para a população mundial. O tratamento envolve medidas farmacológicas e não farmacológicas, como a adoção de um estilo de vida saudável, designadamente a alimentação equilibrada, a diminuição do consumo de álcool, a cessação tabágica e o exercício físico regular.

Para o sucesso do tratamento, é fundamental que haja adesão à terapêutica, existido diversos fatores que a podem influenciar.

A intervenção do Enfermeiro desempenha um papel preponderante, no planeamento em saúde, assim, deve fazer-se a avaliação do estado de saúde de uma comunidade através do conhecimento da sua adesão à 
terapêutica e do seu estilo de vida. O conhecimento destes ajudará o Enfermeiro no desenvolvimento de estratégias promotoras da adesão à terapêutica e a um estilo da vida saudável, da população e da comunidade em geral.

Tendo em conta que $11,8 \%$ dos clientes não aderem à terapêutica, torna-se fundamental que o Enfermeiro continue a desenvolver estas estratégias e ações educativas na comunidade, de forma a aumentar a literacia e capacitar o indivíduo/ família e comunidade, para uma maior adesão à terapêutica e a um estilo de vida saudável.

Os resultados sugerem a necessidade de intervir na promoção da saúde através de um programa comunitário que vise a adesão à terapêutica e capacite a pessoa na adoção de um estilo de vida saudável. Neste âmbito, os enfermeiros, enquanto profissionais de saúde mais próximos da população têm um papel fundamental e determinante.

Como futura linha de investigação sugere-se que seja feito um estudo comparativo na comunidade, com duas amostras de clientes: os que são seguidos e os que não são seguidos em consultas de HTA, na mesma UCSP, com o intuito de avaliar a adesão à terapêutica e o estilo de vida.

\section{Referências}

1. Vilaça AF, Vieira A, Fernandes A, Esteves I, Bouça J, Peixoto V. Disfunção sexual feminina e hipertensão arterial numa população dos cuidados de saúde primários. Revista Portuguesa de Hipertensão e Risco Cardiovascular. 2018;61:6-10.

2. Cairrão AJN, Pinto ASS, Gomes FJP, Marques MAC. Diagnóstico de Situação de Saúde da Cova da Beira. Trabalho apresentado no estágio I, do Mestrado em Enfermagem Comunitária na Escola Superior de Saúde do Instituto Politécnico da Guarda, Portugal; 2018.

3. Direção-Geral da Saúde (DGS). Norma n.o 017/2013 - Avaliação antropométrica no adulto [Internet]. Lisboa: Ministério da Saúde; 2013 [acesso em 14 mai 2018]. Disponível em: http://nocs.pt/wp-content/uploads/2016/06/i019598.pdf

4. World Health Organization (WHO). Hearts, technical package for cardiovascular disease management in primary health care [Internet]. Genebra: WHO; 2016 [acesso em 14 mai 2018]. Disponível em: https://www.who.int/cardiovascular_diseases/hearts/Hearts_package.pdf

5. Martins AJC, Martins JP, Santos SAS. Adherence to the medication regimen before and after a therapeutic awareness-raising intervention. Revista de Enfermagem Referência. 2017;4(14):9-16. DOI:10.12707/RIV17021

6. Ferrão ÂFM. Avaliação dos níveis de adesão ao tratamento e de literacia em saúde numa amostra de doentes com hipertensão arterial. Dissertação de Mestrado apresentada na Universidade da Beira Interior, Covilhã; 2018.

7. Sousa RC, Lucena ALF, Nascimento WS, et al. Particularities of hypertensive elderly people to medicinal treatment adherence. Journal of Nursing UFPE. 2018;12(1):216-223. DOI: 10.5205/1981-8963-v12i01a23296p216-223-2018

8. Ehwarieme TA, Chukwuyem EN, Osayande CO. Knowledge of and compliance with therapeutic regimens among hypertensive patients in Nigeria. Africa Journal of Nursing and Midwifery. 2018;20(1):1-23. DOI:10.25159/2520-5293/3737

9. Ashoorkhani M, Majdzadeh, R, Gholami J, Eftekhar H, Bozorgi A. Understanding non-adherence to treatment in hypertension: a qualitative study. International Journal of Community Based Nursing and Midwifery [Internet]. 2018 [acesso em 14 mai 2020];6(4):314323. https://www.ncbi.nlm.nih.gov/pmc/articles/PMC6226612/pdf/IJCBNM-6-314.pdf

10. Silva TJA, Almeida EUA, Cabral SAAO, Figueiredo CHA. Dificuldades enfrentadas pelo portador de hipertensão arterial sistémica à adesão ao tratamento na Estratégia Saúde da Família. Revista Brasileira de Educação e Saúde. 2017;7(1):23-30. DOI: 10.18378/REBES.V7I1.4839

11. Blanski CRK, Lenardt MH. Compreensão da terapêutica medicamentosa pelo idoso. Revista Gaúcha Enfermagem. 2018;26(2):180-188.

12. World Health Organization (WHO). Adherence to long term therapies. Evidence for action [Internet] Genebra: WHO; 2003 ácesso em 16 jan 2019]. Disponível em: http://apps.who.int/iris/bitstream/10665/42682/1/9241545992.pdf

13. Martins L, Narkiewicz K. Eficácia das associações fixas com perindopril no tratamento da hipertensão arterial, 110 congresso de hipertensão e risco cardiovascular global. Revista Portuguesa de Hipertensão e Risco Cardiovascular. 2017;34:28-30.

14. Cockerham W. Health lifestyle theory and the convergence of agency and structure. Journal of Health Social Behavior. 2005;46(1):51-67. DOI: $10.1177 / 002214650504600105$

15. Direção-Geral da Saúde (DGS). Norma n.o 020/2011 - Hipertensão arterial: definição e classificação [Internet]. Lisboa: Ministério da Saúde; 2013 [acesso em 14 mai 2018]. Disponível em: https://normas.dgs.min-saude.pt/wp-content/uploads/2019/09/hipertensaoarterial_definicao-e-classificacao.pdf

16. Delgado AB, Lima ML. Contributo para a validação concorrente de uma medida de adesão aos tratamentos. Psicologia: Saúde e Doenças. 2001;1:81-100.

17. Marques A, Lúzio F, Martins J, Vaquinhas M. Hábitos alimentares: validação de uma escala para a população portuguesa. Escola Anna Nery Revista de Enfermagem. 2011;15(2):402-409. DOI:10.1590/\$1414-81452011000200025

18. Direção-Geral da Saúde (DGS). Norma n.o 30/2012 - Deteção precoce e intervenção breve no consumo excessivo de álcool [Internet]. Lisboa: Ministério da Saúde; 2012 [acesso em 14 mai 2018]. Disponível em: https://www.dgs.pt/directrizes-da-dgs/normas-e-circularesnormativas/norma-n-0302012-de-28122012-png.aspx

19. Campaniço HMPG. Validade simultânea do questionário internacional de atividade física através da medição objetiva da atividade física por actigrafia proporcional. Dissertação de Mestrado apresentada na Universidade de Lisboa, Faculdade de Motricidade Humana, Lisboa, Portugal; 2016.

20. Direção-Geral da Saúde (DGS). Programa Nacional para a Promoção da Alimentação Saudável - PNPAS. Lisboa: Ministério da Saúde: 2017 [acesso em 14 mai 2018]. Disponível em: https://www.sns.gov.pt/wp-content/uploads/2017/07/DGS_PNPAS2017_V7.pdf

21. Ferreira $P$, Quintal $C$, Lopes $I$, Taveira N. Teste de dependência à nicotina: validação linguística e psicométrica do teste de Fagerström. Dependência Tabágica [Internet]. 2009 [acesso em 14 mai 2019];27(2):37-56. http://hdl.handle.net/10316/13654

22. Afonso AFB. Promover o autocuidado da pessoa com hipertensão arterial. Dissertação de Mestrado apresentada na Escola Superior de Enfermagem de Lisboa, Portugal; 2018. 